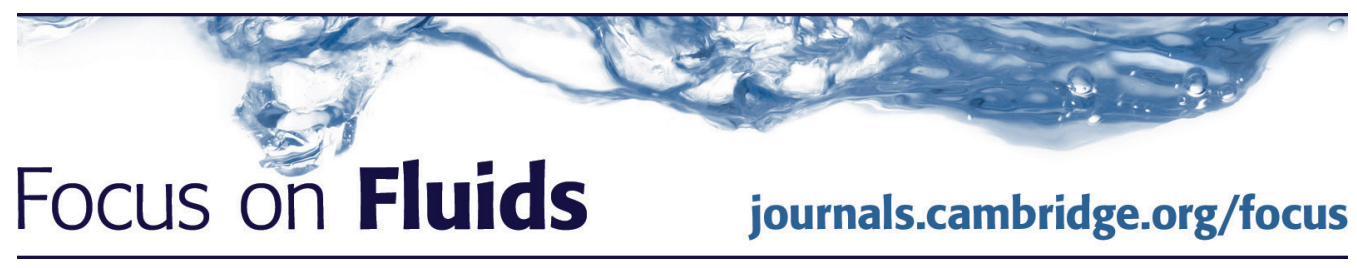

\title{
Suspension dynamics: moving beyond steady
}

\author{
Jason E. Butler $\dagger$ \\ Department of Chemical Engineering, \\ University of Florida, Gainesville, FL 32611, USA
}

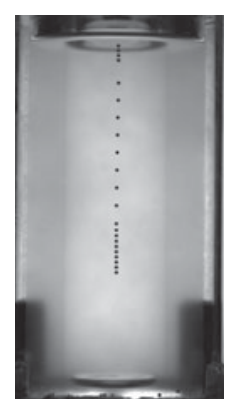

The dynamics of flowing, concentrated suspensions of non-colloidal particles continues to surprise, despite decades of work and the widespread importance of suspension transport properties to industrial processes and natural phenomena. Blanc, Lemaire \& Peters (J. Fluid Mech., 2014, vol. 746, R4) report a striking example. They probed the time-dependent dynamics of concentrated suspensions of rigid and neutrally buoyant spheres by simultaneously measuring the oscillatory rheology and the sedimentation rate of a falling ball. The sedimentation velocity of the ball through the suspension depends strongly on the frequency of oscillation, though the rheology was found to be independent of frequency. The results demonstrate the complexities of suspension flows and highlight opportunities for improving models by exploring suspension dynamics and rheology over a wide range of conditions, beyond steady and unidirectional ones.

Key words: particle/fluid flow, rheology, suspensions

\section{Introduction}

Suspending large rigid particles at high concentrations in a viscous Newtonian fluid creates a suspension that can exhibit complex dynamics and rheology. One might assume that these suspensions would be rather straightforward to model: particle sizes greater than $10 \mu \mathrm{m}$ are sufficiently large that colloidal forces, such as Brownian motion, are negligible, and rigidity negates the need to consider factors such as surface tension or particle elasticity. Many detailed studies of suspensions restrict the problem further, by considering neutrally buoyant particles, often spherical and of a single size, and limiting the flow to viscous conditions. Still, these fluids can exhibit non-Newtonian and nonlinear behaviours, as convincingly demonstrated by Blanc, Lemaire \& Peters (2014) in a simple, and surprising, experiment.

Blanc et al. (2014) probed the rheology and dynamics of concentrated spheres in a Newtonian fluid meeting all of the above restrictions. Limited aspects of these

$†$ Email address for correspondence: butler@che.ufl.edu 
types of suspensions are quasi-Newtonian, including the steady shear rheology. The effective viscosity of a suspension of spheres depends strongly on the volume fraction and is largely independent of the rate of shear, in contrast to the rheology of colloidal, hard spheres (Morris 2009). Accurate evaluation of the effective viscosity for concentrated suspensions is frustrated by a number of difficulties, including the tendency of particles to migrate during viscometric testing (Acrivos 1995). Shear-induced migration occurs in a wide variety of flows other than viscometric ones, including the well-studied case of pressure-driven flows. These shearinduced migrations are argued to result from normal stress imbalances according to macroscopic, multiphase models (Nott \& Brady 1994). Indeed, concentrated suspensions of spheres have measurable normal stress differences, a notable non-Newtonian characteristic (Hinch 2011).

Although modelling at macroscopic scales remains challenging (Nott, Guazzelli \& Pouliquen 2011), Stokesian dynamics simulations (Brady \& Bossis 1988) have provided a number of insights into suspension rheology. These simulations solve for the motion of individual particles from force and torque balances that consider the hydrodynamic forces and the multibody disturbances to the fluid velocity. Typically, the force balances ignore inertia and are supplemented with a short-ranged force to prevent overlap of particle pairs. Calculations for steady shearing flows show the relationship between the structural arrangement of the particles, or microstructure, and the stresses; for example, asymmetries in the distribution of particles are predicted to cause normal stress differences.

Performing unsteady rheological experiments can demonstrate additional, nonNewtonian characteristics of suspensions which arise from the time-dependent coupling between the microstructure and the flow, such as direction-dependent stress responses (Gadala-Maria \& Acrivos 1980). Reversing the direction of shear after attainment of a steady shear-rheology produces a transient stress response as the microstructure, which is asymmetric with respect to the direction of flow, readjusts to the change. Note that the stress, upon stopping the shear flow and then continuing in the same direction, remains constant. The lack of a transient when stopping and then restarting the shear flow in the same direction is due to the absence of forces beyond hydrodynamic ones. Upon cessation of the shearing flow, the particle motion also stops, and when flow is restarted in the same direction, the microstructure already corresponds to that of the steady flow. Investigations of the unsteady motion of suspensions, such as the studies on shear-reversal, are relatively few in number as compared to investigations of steady, unidirectional flows. As a result, the ability to anticipate phenomena in more general flows remains limited, as demonstrated by Blanc et al. (2014).

\section{Overview}

In their investigation of the unsteady flow of suspensions, Blanc et al. (2014) probed the dynamics using two distinct methods. In the first method, the suspension stress was measured in a Couette cell with an imposed oscillatory shear of low frequency and amplitude. In the second, the sedimentation velocity of a heavy ball, much larger than the neutrally buoyant spheres making up the suspension, was recorded. Each measurement, when operated independently, gave results worthy of comment, but the results are of particular interest when the falling ball experiment was performed while also oscillating the suspension. In this latter version of the experiment, the ball sediments orthogonally to the shearing flow. 
First consider the results for a viscous Newtonian fluid in which no particles are present. The stress measurement will be in phase with the oscillatory shear, and the proportionality constant between the instantaneous rate of shear and the instantaneous stress is simply the viscosity. The velocity of the ball falling through the Newtonian fluid is inversely proportional to the viscosity, as predicted by Stokes' law. Since the fluid is viscous and governed by Stokes' equation, the motion of the shearing flow and the sedimentation of the sphere can be superposed. Hence, the sedimentation rate of the falling ball is unaffected by the presence of the oscillatory shearing flow.

Adding the neutrally buoyant and non-colloidal spheres to the fluid at high concentrations qualitatively changes the results. For the rheological measurements, Blanc et al. (2014) found that the response of the suspension is primarily viscous for the range of frequencies and amplitudes tested, as the measured stresses were, except for small deviations, in phase with the instantaneous rate of shear, as would be expected for a Newtonian fluid. Furthermore, the complex viscosity (or just effective viscosity for simplicity) is independent of the frequency. However, the effective viscosity does depend on the strain dependence is due to the self-organization of the particles, which results in different microstructures for different strain amplitudes (Bricker \& Butler 2007).

The falling ball experiment was performed on the suspension in the absence of shear, where the suspension was initially prepared by shearing under either steady or oscillatory conditions to generate different microstructures. The sedimentation velocity of the ball was found to depend only weakly $(5-10 \%)$ on the state of the suspension, even though the effective viscosities for steady shear and oscillatory shear differ by factors of up to eight, depending on the volume fraction of particles in the suspension. In other words, the falling ball is a relatively insensitive probe of the effective viscosity, and associated microstructure, of the suspension. The velocity of the falling ball, however, can be indicative of the frequency of oscillation, even though the measurement of the complex viscosity is not. Blanc et al. (2014) tracked the position of the heavy ball while simultaneously oscillating the suspension and found that the ball can sediment as much as $80 \%$ faster than when falling through the quiescent suspension. The sedimentation rate of the ball when oscillating depends on the strain amplitude and frequency (see figures $4 a$ and $5 a$ ). At any frequency, the maximum velocity of the ball occurs at a strain amplitude of approximately 0.1 or 0.2 . The velocity declines to the rest value as the strain amplitude goes to 1 and exhibits little or no dependence on frequency as the strain amplitude approaches and drops below $10^{-3}$.

The falling ball introduces a second time scale, in addition to the frequency of the oscillation, when the oscillating motion is imposed simultaneously with the sedimentation experiment. Blanc et al. (2014) build a model for the effective viscosity that accounts for the relative importance of the two time scales in controlling the organization of the microstructure created by the shearing flow and its disruption by the falling ball. The model successfully predicts the rate of sedimentation of the falling ball at lower strain amplitudes, but fails to capture the decline in the sedimentation rate of the falling ball that is observed for strain amplitudes larger than 0.1 or 0.2 . The authors note that the onset of the decline in the velocity corresponds to the point at which measurements (by Lin, Phan-Thien \& Khoo 2013) indicate that the oscillatory shear becomes less efficient, or slower, at organizing the microstructure. Hence, the sedimentation of the falling ball, rather than the shearing flow, controls the microstructure, and the ball's velocity returns toward the value corresponding to the suspension at rest. 


\section{Future}

Simulations will, no doubt, be performed on this intriguing set of experiments presented by Blanc et al. (2014). The Stokesian dynamics method, or a similar technique, has the capability to further elucidate the interplay of time scales, forcings and microstructure that creates the observed dynamics. More generally, these results demonstrate that the time-varying flows of suspensions can exhibit significant complexities, despite the seemingly simple constituents of a Newtonian fluid and non-colloidal particles. The complex dynamics arise from the dependence of the flow on the spatial distribution and microstructure of the suspended particles, which, in turn, depends upon the flow field.

Few results on the dynamics of suspensions are available for flows that are not steady and unidirectional; yet the work of Blanc et al. (2014) clearly indicates that much can be learned from exploring a wider range of conditions. Fascinating dynamics can also be observed for suspensions that have slightly different properties than those discussed here; for example, suspensions of non-colloidal rods in Newtonian fluids exhibit unexpected orientational dynamics in oscillatory flows (Franceschini et al. 2011). Further pursuit of the results of Blanc et al. (2014), and other similar investigations, will assist in the refinement of macroscopic equations that can accurately predict suspension dynamics over larger time and length scales than is feasible with particulate simulations.

\section{References}

ACRIVOS, A. 1995 Shear-induced particle diffusion in concentrated suspensions of noncolloidal particles. J. Rheol. 39, 813-826; Bingham Award Lecture-1994.

Blanc, F., Lemaire, E. \& Peters, F. 2014 Tunable fall velocity of a dense ball in oscillatory cross-sheared concentrated suspensions. J. Fluid Mech. 746, R4.

Brady, J. F. \& Bossis, G. 1988 Stokesian dynamics. Annu. Rev. Fluid Mech. 20, 111-157.

BRICKER, J. M. \& BUtLER, J. E. 2007 Correlation between stresses and microstructure in concentrated suspensions of non-Brownian spheres subject to unsteady shear flows. J. Rheol. 51, 735-759.

Franceschini, F., Filippidi, E., Guazelli, É. \& Pine, D. J. 2011 Transverse alignment of fibers in a periodically sheared suspension: an absorbing phase transition with a slowly-varying control parameter. Phys. Rev. Lett. 107, 250603.

GAdAla-MARIA, F. \& ACRIVOS, A. 1980 Shear-induced structure in a concentrated suspension of spheres. J. Rheol. 24, 799-814.

Hinch, E. J. 2011 The measurement of suspension rheology. J. Fluid Mech. 686, 1-4.

Lin, Y., Phan-Thien, N. \& Khoo, B. C. 2013 Short-term and long-term irreversibility in particle suspensions undergoing small and large amplitude oscillatory stress. J. Rheol. 57, 1325-1346.

MorRIS, J. F. 2009 A review of microstructure in concentrated suspensions and its implication for rheology and bulk flow. Rheol. Acta 48, 909-923.

NotT, P. R. \& BRADY, J. F. 1994 Pressure-driven flow of suspensions: simulation and theory. J. Fluid Mech. 275, 157-199.

Nott, P. R., Guazzelli, É. \& Pouliquen, O. 2011 The suspension balance model revisited. Phys. Fluids 23, 043304. 